\title{
Cells behaving badly: a theoretical model for the Fas/FasL system in tumour immunology
}

\author{
Steven D. Webb ${ }^{\mathrm{a}, \mathrm{b}, *}$, Jonathan A. Sherratt ${ }^{\mathrm{b}}$, Reginald G. Fish ${ }^{\mathrm{c}}$ \\ a Department of Mathematical Sciences, Loughborough University, Leicestershire, LE11 3TU, UK \\ b Department of Mathematics, Centre for Theoretical Modelling in Medicine, Heriot-Watt University, \\ Edinburgh EH14 4AS, UK \\ ' Cancer Research Wales Laboratories, Velindre Hospital, Whitchurch, Cardiff CF14 2TL, UK
}

Received 24 January 2002; received in revised form 29 May 2002; accepted 7 June 2002

\begin{abstract}
One proposed mechanism of tumour escape from immune surveillance is tumour up-regulation of the cell surface ligand FasL, which can lead to apoptosis of Fas receptor (Fas) positive lymphocytes. Based upon this 'counterattack', we have developed a mathematical model involving tumour cell-lymphocyte interaction, cell surface expression of Fas/FasL, and their secreted soluble forms. The model predicts that (a) the production of soluble forms of Fas and FasL will lead to the down-regulation of the immune response; (b) matrix metalloproteinase (MMP) inactivation should lead to increased membrane FasL and result in a higher rate of Fas-mediated apoptosis for lymphocytes than for tumour cells. Recent studies on cancer patients lend support for these predictions. The clinical implications are two-fold. Firstly, the use of broad spectrum MMP inhibitors as anti-angiogenic agents may be compromised by their adverse effect on tumour FasL up-regulation. Also, Fas/FasL interactions may have an impact on the outcome of numerous ongoing immunotherapeutic trials since the final common pathway of all these approaches is the transduction of death signals within the tumour cell.
\end{abstract}

(C) 2002 Published by Elsevier Science Inc.

Keywords: Modelling; Cancer cells; FasL; Apoptosis; Immune evasion

\footnotetext{
${ }^{*}$ Corresponding author. Tel.: +44-1509 228 203; fax: +44-1509 223969.

E-mail address: s.d.webb@lboro.ac.uk (S.D. Webb).
} 


\section{Nomenclature}

TGF $\beta$ type $\beta$ transforming growth factor

IL-10 interleukin 10

TNF tumour necrosis factor

AICD activation-induced cell death

MMP matrix metalloproteinase

Fas Fas receptor

FasL Fas ligand

sFasL soluble Fas ligand

sFas soluble Fas receptor

MMPI matrix metalloproteinase inhibitor

\section{Introduction}

Although virally infected cells may produce 'danger' signals that can lead to specific lymphocyte recognition, many human solid tumours are only weakly immunogenic [1]. This phenomenon has led to the use of identified tumour-associated antigens as vaccines in numerous clinical trials, and to intense study of the mechanisms involved in immune cell recognition. For simplicity, the specific interactions of lymphocytes with target cells may be divided into two basic, overlapping stages, (a) cell-cell recognition and interaction (reviewed in [2]) followed by (b) lymphocytemediated killing of target cells by apoptosis (reviewed in [3]). However, some virally-infected cells and tumour cells have, not surprisingly, developed various strategies to escape from the action of immune cells at both of these stages.

In the first stage, tumour cells not only use mechanisms that prevent recognition by lymphocytes, such as down-regulation of MHC class I molecules and co-stimulatory signals like B7, but also some tumours actively contribute to impairment of lymphocyte function by secreting factors with immunosuppressive activity (e.g. TGF $\beta^{3}$ or IL-10) and altering lymphocyte signalling [4]. Attempts to correct one or more of these defects in cancer patients have led to numerous immunotherapeutic trials, but the clinical results so far have been rather disappointing. There is, therefore, a clear need to further understand the mechanisms of tumour cell-lymphocyte interactions and to develop new approaches to therapy in this field.

It is known that tumour cells have developed several other escape strategies involving the killing mechanisms. In particular, some tumour cells have been shown to become more resistant to apoptosis via alterations in the apoptotic signal transduction pathways [5]. Another possible mechanism is that tumour cells can kill activated lymphocytes through tumour cell production of the death ligand, FasL, a phenomenon known as the tumour FasL counterattack model [6]. FasL and its receptor, Fas (also termed APO-1, CD95) are membrane-bound glycoproteins and are members of the tumour necrosis factor (TNF) family. A FasL expressing cell, upon interaction with a Fas bearing cell, can transmit an apoptotic signal and the latter cell can undergo programmed cell death. In the immune system, the Fas/FasL signalling pathway appears to be in- 
volved in the peripheral deletion of self-reacting lymphocytes, in killing by cytotoxic T-cells, in terminating an immune reaction through elimination of activated lymphocytes after an immune response (AICD), and in maintaining immune privilege in certain normal tissues, such as the eye and testis, through epithelial cell expression of FasL [7,8]. One important regulatory control for membrane-bound FasL is its cleavage to a smaller, relatively less cytotoxic, soluble form (sFasL) by matrix metalloproteinase (MMPs) in the microenvironment, and certain cells can also secrete a soluble form of Fas, not by MMP cleavage, but by alternate splicing of Fas mRNA transcripts. Both these strategies can lead to down-regulation of the immune response $[9,10]$.

Lymphocytes constitutively express the Fas receptor and upon specific interaction with virallyinfected cells or tumour cells, the activated lymphocytes induce the cell surface expression of FasL. Coupling with Fas positive tumour cells can lead to tumour cell apoptosis. It is now known that a number of human tumour cell types can express both mRNA transcripts and membranebound FasL protein, and often down-regulate cell surface Fas [11,12]. Moreover, the soluble forms of receptor and ligand have been shown to be produced by some tumour cells in vitro and these soluble molecules are often elevated in the serum of patients with a number of histological tumour cell types [13-15]. The sFasL can bind to Fas and the complex formed is rapidly internalised [15], resulting in downregulation of the receptor. The soluble ligand can also inhibit the cytotoxicity of FasL $[15,16]$. These results suggest that the soluble form of the ligand may play an important role in the regulation of Fas-mediated apoptosis.

Tumour-immune cell dynamics have been investigated in several theoretical studies (reviewed by Adam and Bellomo [17]). Several authors have incorporated growth factors and modulators into models of the immune response to cancer growth [18-20]. A model for the spatial interactions of tumour associated macrophages, normal cells and tumour cells, has been developed, which suggests that tumour heterogeneity may arise as a natural consequence of macrophage infiltration [21]. The effects of vascularization on tumour-immune system interactions within a multicellular tumour spheroid have also been considered [22], as well as a model of natural killer cell recognition of target cells based on cell adhesion and the delivery of cytotoxic hits [23]. More recently, Fas/FasL has been investigated in a model for cytokine-modulated Th1/Th2 helper T-cell differentiation [24]. In this study, the Fas/FasL system is shown to be an important factor in stabilizing the two polarized arms of the Thl/Th2 T helper population in response to a pathogenic challenge.

Here, we develop a new model for the Fas/FasL system to investigate the role of Fas/FasL in immunosuppression induced by human tumours. We show that the constitutive expression of FasL by tumour cells is a potential mechanism of immune evasion, and our model predicts that sFasL acts as an inhibitor to the Fas death signal. In addition, we investigate the enzymatic cleavage of FasL into sFasL. The model predicts that inhibition of MMP activity causes Fas sensitive lymphocytes to undergo Fas-mediated apoptosis more rapidly than Fas-resistant tumour cells.

\section{Development of a mathematical model}

The model consists of ordinary differential equations which represent the crosslinking of FasL and Fas, with one set of these equations for each cell. We consider the interactions of two cell types: armed effector T-cells, and FasL positive tumour cells. The model is based upon the demonstration that certain types of human tumours can produce functional FasL and can induce 
the apoptotic killing of activated lymphocytes in vitro. Our model consists of a series of equations for tumour cells $(m)$, T-cells $(T)$, soluble Fas ligand $\left(S_{L}\right)$, and numbers of Fas ligand molecules $\left(L_{m}\right.$, $\left.L_{T}\right)$ and unoccupied Fas receptors $\left(R_{m}, R_{T}\right)$ on the surface of each cell. Subscripts $m$ and $T$ are used to denote the number of ligand or receptor molecules on each tumour cell and T-cell, respectively. With this notation, the model system is as follows:

$$
\begin{aligned}
& T=\text { T-cells: } \quad \frac{\mathrm{d} T}{\mathrm{~d} t}=\overbrace{-k_{1} m T L_{m} R_{T}}^{\mathrm{T} \text {-cell killing }} \\
& L_{T}=\text { T-cell FasL: } \quad \frac{\mathrm{d} L_{T}}{\mathrm{~d} t}=\overbrace{k_{2} T m}^{\text {T-cell FasL production }}-\underbrace{k_{3} L_{T}}_{\text {MMP cleavage }}-k_{4} L_{T}-\overbrace{k_{5} m T L_{T} R_{m}}^{\text {killing down-regulation }} \\
& R_{T}=\text { T-cell Fas: } \quad \frac{\mathrm{d} R_{T}}{\mathrm{~d} t}=k_{6}-k_{7} R_{T}-\overbrace{K_{8} R_{T} S_{L}}^{\text {sFasL/Fas complex }} \\
& m=\text { tumour cells: } \quad \frac{\mathrm{d} m}{\mathrm{~d} t}=\overbrace{-k_{1} m T L_{T} R_{m}}^{\text {tumor killing }} \\
& L_{m}=\text { tumour FasL: } \frac{\mathrm{d} L_{m}}{\mathrm{~d} t}=\overbrace{k_{10}}^{\text {tumour FasL production }} \underbrace{-k_{3} L_{m}}_{\text {MMP cleavage }}-k_{4} L_{m}-\overbrace{k_{5} m T L_{m} R_{T}}^{\text {killing down-regulation }} \\
& R_{m}=\text { tumour Fas: } \frac{\mathrm{d} R_{m}}{\mathrm{~d} t}=k_{6}-k_{7} R_{m}-\overbrace{k_{8} R_{m} S_{L}}^{\text {sFas } / \text { Fas complex }} \\
& S_{L}=\text { sFasL: } \quad \frac{\mathrm{d} S_{L}}{\mathrm{~d} t}=\overbrace{k_{3}\left(L_{m} m+L_{T} T\right)}^{\text {FasL cleavage }}-k_{11} S_{L}-\underbrace{k_{9}\left(R_{m} m+R_{T} T\right) S_{L}}_{\text {sFasL/Fas formation }},
\end{aligned}
$$

where the $k_{i}$ s are positive constants. The model is based on the framework illustrated in Fig. 1.

\subsection{Model assumptions}

T-cells must be activated to proliferate and differentiate into cells capable of killing tumour cells. The activation of naive T-cells requires recognition of antigen bound to an MHC molecule, but this is not on its own sufficient for activation; a second signal is required for optimal T-cell growth and differentiation [25]. The second signal involves interactions between a B7 molecule on an antigen presenting cell, or tumour cell, and the B7 receptor on the T-cell [25]. This dual requirement for both antigen:MHC complex recognition and B7 co-stimulation helps to prevent naive T-cells from responding to antigens on self tissue cells. Thus, for a effective immune response, tumour antigen needs to reach the lymphoid tissues and be presented on MHC molecules 


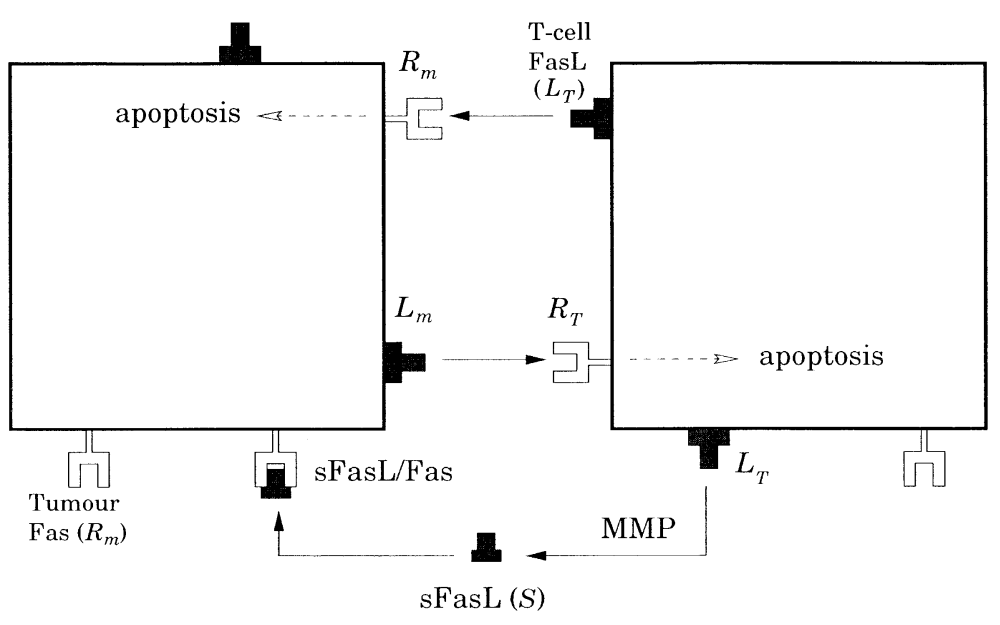

Fig. 1. Schematic representation of the interactions between FasL, soluble FasL, and Fas receptor during an immune attack against a tumour cell. Cytotoxic T-cells recognize tumour cells, and are activated to express FasL. FasL then binds to Fas on the surface of the tumour cells and induces their death through apoptosis. Some tumour cells also express FasL, and T-cells express up-regulated Fas, probably because of activation in response to tumour antigen. This enables the tumour to counterattack the cytotoxic T-cells: FasL expressed on tumour cells binds to Fas on the T-cells, which then die by apoptosis before they can attack the tumour. FasL is cleaved by a MMP to produce the soluble form sFasL. This is functional, and may bind to Fas, forming membrane-bound sFasL/Fas complexes, which are quickly internalized.

expressed on B7-positive antigen presenting cells [26]. The initial contact with antigen and costimulatory factors leads to the production of armed effector T-cells, which can act on any tumour cell that display antigen:MHC molecules on its surface. We choose T-cells from this level of immune response development to be the focus of our model, and we assume that the tumour cell population is antigen: $\mathrm{MHC}^{+}$, so that each effector T-cell can act on any tumour cell. Given that tumour cells have a long half life, a turnover time of days [27], and that the duration of the cell cycle is of the order $18 \mathrm{~h}$ [24], we anticipate that cellular growth terms will be small compared to the time scale of the Fas/FasL reaction, based on the short time frame used in the analysis (the typical time a cytotoxic T-cell can program apoptosis in a target cell is around 5 min [25]).

The terms used for FasL mediated killing of tumour cells and lymphocytes are of the same form, and for simplicity the same rate constant $\left(k_{1}\right)$ is used in both terms. Note that we have omitted details such as: T-cell-to-T-cell apoptosis, where the crosslinking of Fas by FasL present on the Tcells will induce apoptosis, thereby deleting potentially auto-aggressive T-cells following an immune response; and tumour-to-tumour cell apoptosis, where tumour cells expressing both Fas and FasL may induce apoptosis in themselves. We have neglected these types of cell-cell interactions to focus on the battle between the host and tumour cells, and to investigate the role of tumour FasL in the maintenance of immune privilege status within the tumour microenvironment.

We assume that Fas is constitutively expressed on the surface of both lymphocytes and tumour cells. However, since Fas is down-regulated in a number of tumour types and up-regulated in activated lymphocytes, we consider that the inital expression of Fas is significantly higher in 
T-cells than in tumour cells. For simplicity, we use the constant production term $k_{6}$ to represent the delivery of Fas to the cell surface. The number of free Fas receptors decay at the rate $k_{7}$.

Tumour cells, and non-haematopoietic cells which use FasL to maintain immune privilege, express FasL directly on the cell surface [13,28]. In contrast, newly synthesized FasL is stored in specialized lysosomes in T-cells, and is delivered to the cell surface only when the cytotoxic lymphocytes recognize target cells $[29,30]$. This targeting to secretory lysosomes of T-cells provides a mechanism for controlling the cell surface appearance of FasL. Based on these observations, we use a term proportional to the tumour and T-cell populations to represent the production of FasL to the surface of T-cells; this is the $k_{2} \mathrm{Tm}$ term. The constitutive expression of FasL on the surface of tumour cells is given by $k_{10}$; that is, it is supplied at a constant rate. We assume both tumour and T-cell degrade linearly proportional to their concentrations (at a rate given by $k_{4}$ ). FasL is used up in the binding to Fas at a rate directly proportional to the rate of cell killing; these are the $k_{5} m T L_{T} R_{m}$ and $k_{5} m T L_{m} R_{T}$ terms for tumour and T-cell FasL, respectively.

To keep our model as simple as possible, we do not consider MMPs explicitly. Instead, the simple linear terms $k_{3} L_{T}$ and $k_{3} L_{m}$ represent the cleavage of FasL by MMPs from the surface of Tcells and tumour cells, respectively. The concentration of sFasL decays at the rate $k_{11}$. We also assume that a sFasL molecule binds irreversibly to a Fas receptor on the cell surface. The terms $k_{8} R_{T} S_{L}$, and $k_{8} R_{m} S_{L}$ represent the formation of $\mathrm{sFasL} / \mathrm{Fas}$ complexes on the surface of T-cells and tumour cells, respectively.

To numerically simulate the apoptotic killing of tumour cells and activated T-cells in vitro, we use initial conditions corresponding to the down-regulation of the Fas receptor and the constitutive expression of FasL in tumour cells. We also assume that activated tumour infiltrating T-cells initially express up-regulated levels of the Fas receptor compared to tumour cells, as observed in tumour-infiltrating lymphocytes from renal cell carcinoma patients. sFasL is initially absent from the culture medium. Note that the number of T-cells $(T)$ used in the standard cytotoxicity assay always exceeds that of tumour targets $(m)$. However, the $T: m$ ratio in any solid tumour in vivo is almost invariably in favour of the tumour cells, even when considerable numbers of tumour infiltrating T-cells are present [31]. This structure creates a T-cell gradient extending from the medium boundary to the tumour-immune interface. Biologically, it is the region where the number of T-cells is the same as the targeted tumour cells, where the $T: m$ ratio is one. For a growing tumour escaping immune control, we expect to see this region advancing towards the medium boundary, with receding waves of T-cells. In our model, we focus our attention on the evolution of a $T: m=1$ state by imposing initial conditions such that the number of T-cells and tumour cell targets are equal.

\subsection{Parameter values}

At present, there is still a lack of quantitative data for Fas/FasL. However, Fas/FasL is in the same family as TNF/TNFR [32], and the extracellular domains of members of the TNF family are known to be well conserved, suggesting that values for Fas/FasL should be at least of the same order of magnitude as for TNF/TNFR. The receptor/ligand binding parameters for TNF are: number of receptors per cell, $R=6.6 \times 10^{3}$ molecule/cell; binding, $k_{\text {bind }}=9.6 \times 10^{8} \mathrm{M}^{-1} \mathrm{~min}^{-1}$; and dissociation, $k_{\text {diss }}=0.14 \mathrm{~min}^{-1}$ [33].

Assuming that soluble Fas binds to the cell surface Fas molecule at a similar rate to that of TNF binding [32], we set $k_{8}$ and $k_{9}$ to be the same as $k_{\text {bind }}=9.6 \times 10^{8} \mathrm{M}^{-1} \mathrm{~min}^{-1}$. Experimental 
work of Watanabe-Fukunaga et al. [34] shows that the half-life of the TNF receptor is around $2 \mathrm{~h}$, which yields $k_{7}=\ln (2) / 2 \mathrm{~h}^{-1} \approx 0.34 \mathrm{~h}^{-1}$.

In the complete absence of any binding, no T-cell FasL will be produced, but there will be a background level of Fas receptor and tumour FasL expression, say $R_{\text {cell }}$ and $L_{\text {cell }}$ respectively. This is the trivial steady state of the model, and so the equations for $R_{T}, R_{m}$ (1c) and (1f) and $L_{m}$ (1e) gives $k_{6}=k_{7} R_{\text {cell }}$ and $k_{10}=\left(k_{3}+k_{4}\right) \mathrm{L}_{\text {cell }}$, respectively. Here, we make use of the data for TNF binding and take $R_{\text {cell }}=R=6.6 \times 10^{3}$ [32], giving $k_{6}=k_{7} R_{\text {cell }}=0.34 \times 6.6 \times 10^{3} \mathrm{cell} / \mathrm{ml} \mathrm{h}^{-1}$.

We leave the parameter $\left(k_{3}\right)$ to be varied in model simulations to represent different levels of MMP inhibition. The range of values taken for $k_{3}$ is $0.693-15 \mathrm{~h}^{-1}$, which corresponds to a half-life FasL turnover of $60 \mathrm{~min}$ decreasing to $3 \mathrm{~min}$. If we take $k_{3}=0.693 \mathrm{~h}^{-1}$ and assume that the unstimulated tumour FasL number $L_{\text {cell }}$ is 3000 molecule/cell, then this gives the estimate $\left(k_{3}+k_{4}\right) \mathrm{L}_{\text {cell }}=3.11 \times 10^{3}$ molecule/cell $\mathrm{h}^{-1}$ for $k_{10}$. We are unaware of any data on the decay rate of membrane-FasL, but we estimate that this will be similar to that of the turnover of TNF receptors, giving $k_{4}=0.35 \mathrm{~h}^{-1}$.

Studies have shown that when cytotoxic T-cells are mixed with target cells, they can program specific target cells to undergo apoptosis within $5 \mathrm{~min}$, although death may take hours to become fully evident [25]. We approximate the FasL-mediated cell killing $\left(k_{1}\right)$ by the apoptotic signalling of target cells in CTL/target clusters, giving $k_{1}=8.38 \times 10^{-10}$ cell $/ \mathrm{ml}$ molecule ${ }^{-2} \mathrm{~h}^{-1}$ and $k_{5}=2.52 \times 10^{-9} \mathrm{ml}^{2} /$ cell molecule ${ }^{-1} \mathrm{~h}^{-1}$. Our estimate for $k_{2}$, the rate constant controlling the release of FasL to the surface of T-cells following activation by antigen, is taken from a study of Kiener et al. [35]. In this study, the stimulation of monocytic cells with antigen gave rise to the rapid release of FasL to the cell surface, within $15 \mathrm{~min}$, and was dependent on the concentration of stimulatory agent. This gives $k_{2}=6 \times 10^{-3}$ molecule $\mathrm{ml}^{2}$ cell ${ }^{-3} \mathrm{~h}^{-1}$. We are not aware of any quantitative measurements for the half-life of sFasL. However, polypeptides such as interleukin-2, when injected into the blood stream of patients, decline with a half-life of several minutes [33]. Hence, we take $k_{11}=13.9 \mathrm{~h}^{-1}$ for sFasL, corresponding to a half-life of $3 \mathrm{~min}$.

\subsection{Steady states}

Non-negative steady states of (1a)-(1g) are found by setting the time derivatives to zero and solving the resulting system of algebraic equations. We divide the steady states into three categories:

(i) trivial state, where both the population of T-cells and tumour cells are zero, given by

$$
T=0, \quad m=0, \quad L_{T}=0, \quad L_{m}=k_{10} /\left(k_{3}+k_{4}\right), \quad R_{T}=R_{m}=k_{6} / k_{7}, \quad S_{L}=0
$$

(ii) normal immune state, with T-cells and no tumour cells, with

$$
T=T^{*}, \quad m=0, \quad L_{T}=0, \quad L_{m}=k_{10} /\left(k_{3}+k_{4}\right), \quad R_{T}=R_{m}=k_{6} / k_{7}, \quad S_{L}=0
$$

(iii) tumour state, with mutant cells and no T-cells, given by

$$
\begin{aligned}
& T=0, \quad L_{T}=0, \quad L_{m}=k_{10} /\left(k_{3}+k_{4}\right), \quad R_{T}=R_{m}=k_{6} /\left(k_{7}+k_{8} S_{L}^{*}\right), \\
& m=\frac{\left(k_{3}+k_{4}\right) k_{11} S_{L}^{*}\left(k_{7}+k_{8} S_{L}^{*}\right)}{k_{3} k_{7} k_{10}+S_{L}^{*}\left(k_{3} k_{8} k_{10}-\left(k_{3}+k_{4}\right) k_{6} k_{9}\right)}, \quad S_{L}=S_{L}^{*} .
\end{aligned}
$$


Note that in the 'trivial' and 'tumour only' steady states, we have $R_{T}>0$ and at the same time $T=0$. To explain this, it is important to emphasise that $R_{T}$ is the number of Fas receptors per Tcell, so denoting the total number of Fas receptors for the entire T-cell population by $R_{T}^{\text {total }}$ yields $R_{T}=R_{T}^{\text {total }} / T$. Thus, there is no contradiction to having $T=0$ but $R_{T}>0$; the latter is simply the limiting number of receptors per cell as the cell number decreases to zero. Similarly, we have $L_{m}>0$ and at the same time $m=0$ in the 'normal immune' steady state.

With $S_{L}^{*}$ the convenient parameter, we see that there is a one parameter family of tumour steady states. This is an important property of the model, since it illustrates an association between an increase in soluble ligand $\left(S_{L}\right)$ and tumour cell density $(m)$. As the concentration of sFasL increases, the steady state level of tumour cells increases, and the number of free receptors on the surface of each cell decreases. A higher tumour cell density means less cell killing, which suggests that $\mathrm{sFasL}$ acts by down-regulating the cytotoxic activity of the membrane-bound Fas ligand. Intuitively, this is because high levels of sFasL will result in the production of large amounts of sFasL/Fas complexes on the cell surface, resulting in a down-regulation of the Fas receptor and consequent inhibition of the cytotoxic activity of FasL. A similar phenomenon is observed when we extend the model to include the presence of the soluble form of Fas receptor. In this case, sFas inhibits the FasL death signal by forming complexes with the membrane-bound ligand, and a correlation between high concentrations of sFas and low FasL mediated apoptosis is observed in the model solutions (data not shown). These results are in agreement with experimental observations (for example, see [10,14-16]), and further highlight the importance of soluble Fas-components in the Fas-counterattack model.

The eigenvalues $\lambda$ of the Jacobian matrix at a fixed point of system (1a)-(1g) determines the stability of the steady state. If each $\operatorname{Re}(\lambda)>0$ then the steady state is unstable while if each $\operatorname{Re}(\lambda)<0$ it is stable. Evaluating the eigenvalues of the Jacobian for each of the steady states (i), (ii) and (iii) shows that in the model system (1a)-(1g) the tumour-only state is stable, while the trivial and T-cell steady states are unstable. This means that, starting with a small population of T-cells and tumour cells, the system will evolve to the tumour only state, leading to clearance of the T-cell population and immune evasion by the tumour cells. This predicts that T-cells armed with FasL are incapable of preventing FasL positive mutant cells escaping immune rejection. This can be seen in Fig. 2 where we present a typical numerical simulation of the model equations. This simulation illustrates the temporal evolution of the model solutions over a period of $24 \mathrm{~h}$. The numbers of ligand and receptor molecules rapidly attain their steady state values. After $24 \mathrm{~h}$, the densities of T-cells and tumour cell targets have evolved so that the T: $m$ ratio is approximately 1:4. As time progresses, the tumour population decreases to its steady state value, and the activated Tcell population decays to zero.

An interesting result is the sharp jump in the concentration of sFasL. In a separate paper [36], we study this behaviour using singular perturbation analysis on a caricature representation of the model system (1a)-(1g). In contrast to a standard matched asymptotic expansion, this problem has an unusual asymptotic structure. Briefly, instead of the usual solution form, with two outer solutions separated by a single transition layer centred at the point at which the sharp jump in the sFasL solution occurs, our solution contains multiple fast time layers, with each layer being necessary to capture the entire dynamics of the sharp transition. This analysis has enabled us to identify the mechanism by which the sharp transition in the sFasL solution is generated; we 

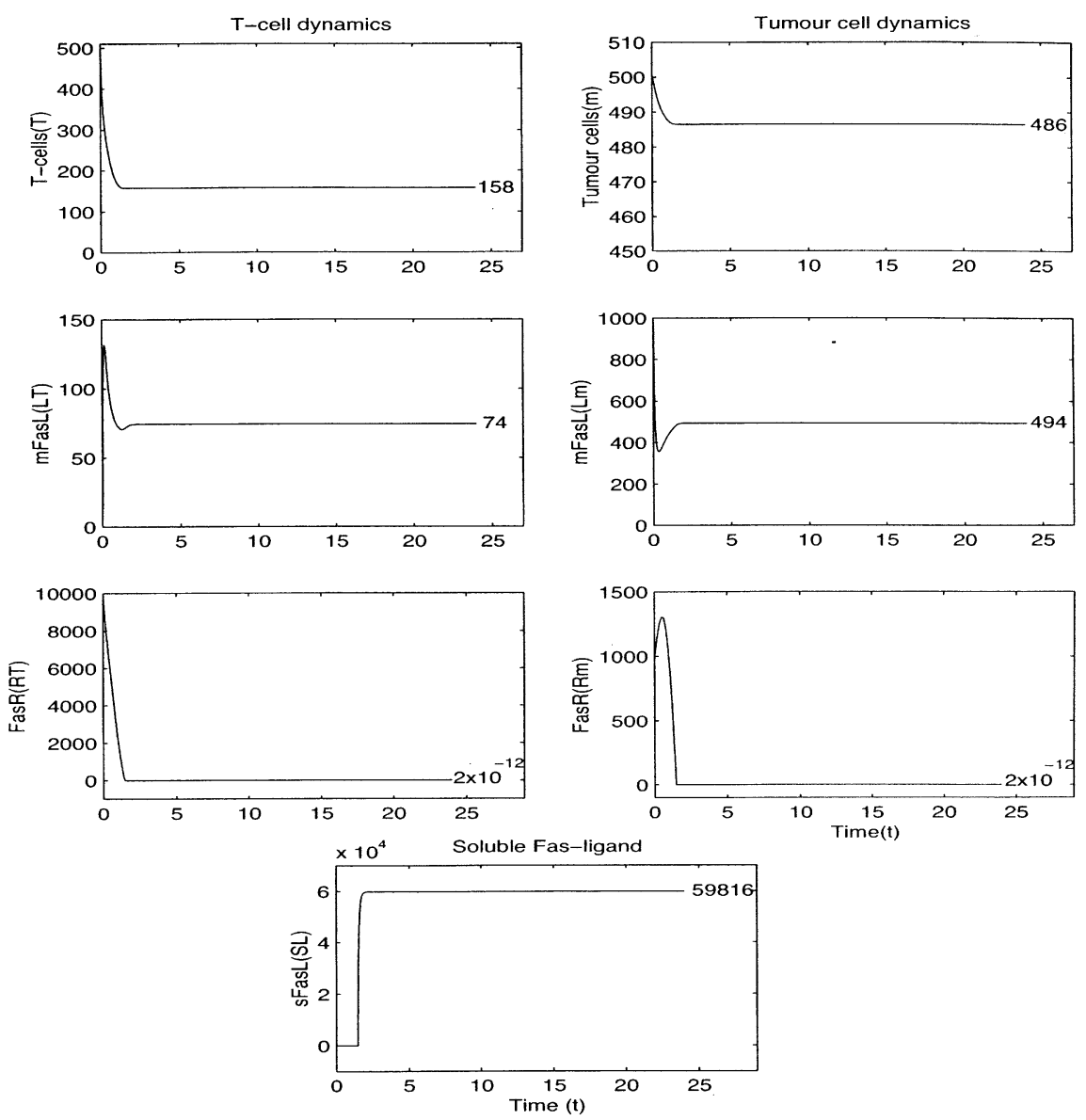

Fig. 2. Typical simulation of the model equations (1a) $-(1 \mathrm{~g})$ over a period of $24 \mathrm{~h}$. We take as initial conditions $T(0)=m(0)=500, S_{L}(0)=L_{T}(0)=0, R_{m}(0)=L_{m}(0)=10^{3}$ molecule $/$ cell, $R_{T}(0)=10^{4}$ molecule $/$ cell. The variable $t$ is time in hours. The decrease in cell number is very slow in comparison to the receptor and ligand dynamics. After $24 \mathrm{~h}$, the densities of T-cells and tumour cells evolve so that their T:m ratio is approximately 1:4. As time progresses, the tumour population decreases to its steady state value $\left(m^{*} \approx 437\right.$ cells $\left./ \mathrm{ml}\right)$, and the T-cell population decays to zero.

predict that the level of sFasL is closely related to the levels of cell membrane Fas, and the jump in sFasL is initiated when Fas falls to very low values. This makes sense intuitively since a low number of free Fas receptors will not be able to bind with the large amounts of sFasL shed from the surface of the cells, resulting in the accumulation of high levels of extracellular soluble ligand.

\subsection{The Fas-counterattack}

In the model, there are two basic differences between tumour cells and T-cells: (i) tumour cells express down-regulated levels of the Fas-receptor compared to T-cells, which up-regulate Fas expression in response to tumour antigen; and (ii) tumour cells constitutively express FasL, 
(a): Fas expression

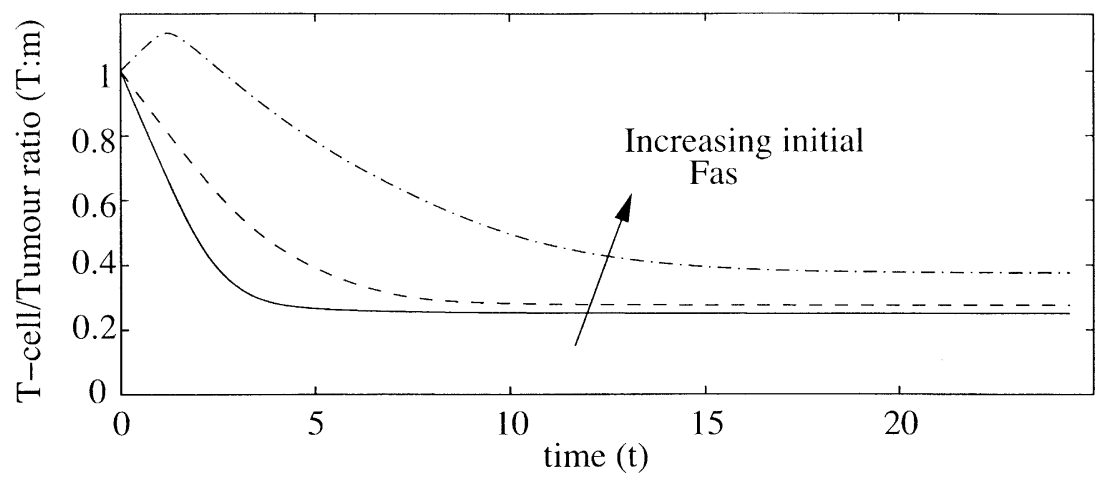

(b): FasL production

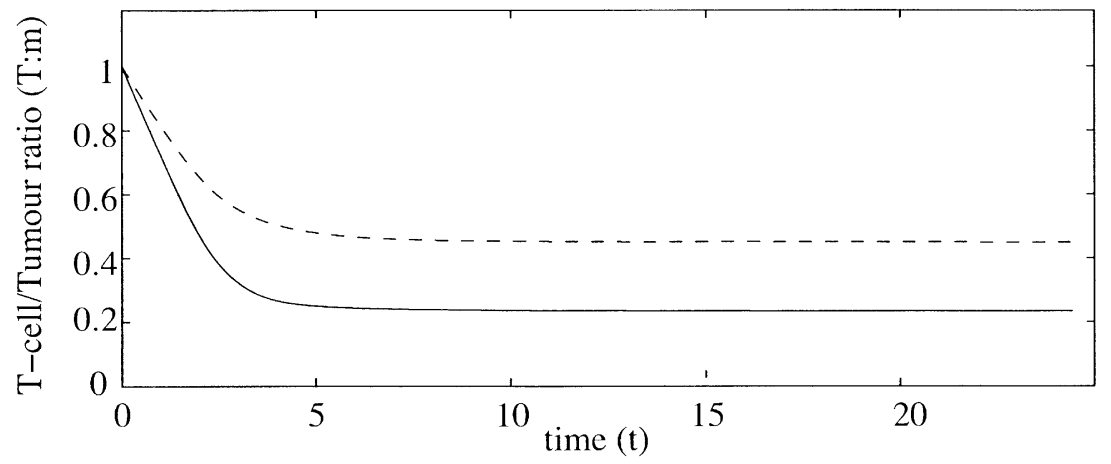

Fig. 3. Temporal evolution of the T-cell/tumour cell ratio (T:m). In (a) we vary $R_{m i}$, the initial number of Fas receptors on the surface of each tumour cell. Increasing $R_{m i}$ increases the sensitivity of tumour cells to the cytotoxic effect of FasL. We observe that the T: $m$ ratio increases as $R_{m i}$ increases. The curves shown represent the $T: m$ ratio for the cells in three cases, in which $R_{T}(0)=10^{4}$ molecule/cell, and $R_{m i}=R_{m}(0)$ has the value (in molecules/cell) (i) $10^{3}$ (-), (ii) $10^{4}(--)$, (iii) $5 \times 10^{4}(--)$. In (b) we show the effect of imposing the constitutive expression of FasL in T-cells. The solid line in (b) is the $T: m$ profile when tumour cells constitutively express FasL, whereas T-cells express FasL only on activation. The dashed line corresponds to both cell types expressing FasL constitutively. The results show that there is a much greater increase in the $T: m$ ratio when FasL is expressed in the same way in both cell types, compared to when Fas expression is up-regulated in tumour cells. The parameter values and initial conditions used in this simulation are the same as in Fig. 2. In (b), to allow the constitutive expression of FasL in T-cells, we replace the term $k_{2} T m$ in the $L_{T}$ equation with the constant $k_{10}=3.11 \times 10^{3}$ molecule cell ${ }^{-1} \mathrm{~h}^{-1}$.

whereas T-cells express FasL only upon activation. In order to establish the relative importance of these mechanisms in maintaining tumour-immune privilege, we have carried out appropriate model simulations.

If we change the initial conditions so that Fas receptor expression is up-regulated in tumour cells, we observe that the cytotoxic activity of the T-cells increases. This is illustrated in Fig. 3(a). Here, we plot the temporal evolution of the T-cell/tumour cell ratio $(T: m)$ for $24 \mathrm{~h}$. In the original simulation (Fig. 2) there is a down-regulation of Fas expression in tumour cells, and the tumour and T-cell densities evolve so that their $T: m$ ratio is approximately 0.24 . As we increase the initial 
level of tumour Fas $\left(R_{m i}\right)$, the $T: m$ ratio increases (Fig. 3(a)). With the initial level of Fas receptor the same for both cell types, the $T: m$ ratio after $24 \mathrm{~h}$ increases to approximately 0.28 . If we vary initial conditions so that tumour cells express up-regulated levels of Fas receptor compared to Tcells, the $T: m$ ratio after $24 \mathrm{~h}$ becomes approximately 0.37 .

In Fig. 3(b), we show the effect of replacing the term $k_{2} T_{m}$ - the term in the T-cell FasL equation controlling FasL production - with $k_{10}$. This has the effect of imposing the constitutive expression of FasL in T-cells. Thus, the production of FasL on the surface of T-cells is the same as that as in tumour cells, and no longer dependent on target cell recognition. Here, we observe that the $T: m$ ratio increases from 0.24 , in the original simulation of Fig. 2, to 0.45 .

In these two cases the final $T: m$ ratio is less than one, implying that both the down-regulation of Fas and the constitutive expression of FasL in tumours play important roles in the Fas counterattack model. However, the results show that there is a much greater increase in the $T: m$ ratio when FasL is expressed constitutively in both cell types compared to when Fas is up-regulated in tumour cells. This suggests that although both mechanisms are significant, alterations in Fas ligand expression by tumour cells is the dominant term, and is the key difference between the two cell types.

\subsection{Effect of MMP inactivation}

To simulate the functional role of a matrix metalloproteinase inhibitor (MMPI), we vary the rate constant $k_{3}$, the constant that controls the MMP-mediated cleavage of FasL into sFasL. Decreasing the value of $k_{3}$ corresponds to an increase in MMPI treatment. If we study the variation of the $T: m$ (T-cell/tumour) ratio with different values of $k_{3}$, with the remaining rate constants and parameters fixed, we see that decreasing $k_{3}$ causes a decrease in the $T: m$ ratio (recall that low $k_{3}$ corresponds to a low rate of MMP cleavage of FasL into sFasL, resulting in reduced levels of sFasL). This is illustrated in Fig. 4. Here we plot the $T: m$ ratio after $48 \mathrm{~h}$ for varying levels of MMPI. As $k_{3}$ increases, we observe that the levels of T-cells, tumour cells and sFasL also increase. The decrease in the $T: m$ ratio with increased MMPI (small $k_{3}$ ) can be explained by Fig. 4(a) and (b): here, we observe that increasing $k_{3}$ has the greatest effect on the level of T-cells. For example, increasing $k_{3}$ from 5.9 to $13.8 \mathrm{~h}^{-1}$, increases the density of T-cells by $69 \%$, and tumour cells by $1.5 \%$ (Fig. 4, solid line). These results indicate that MMP inactivation causes Tcells to undergo apoptosis more rapidly than tumour cells.

In Fig. 4, we show the effects of varying the Fas-sensitivity of the two cell types. If we keep ligand production constant, so that both cell types express FasL constitutively, we observe that the qualitative profile for the $T: m$ ratio against the rate constant $k_{3}$ remains unchanged (i.e. a decrease in the $T: m$ ratio with increasing MMPI). High values of $R_{m i}$, the initial number of free receptors on the surface of each tumour cell, also induce a decrease in the $T: m$ ratio for increased MMPI. In both cases, there is a decrease in the $T: m$ ratio with the addition of MMPI. As a comparison, we include the case where FasL expression and the levels of Fas receptor are the same in both cell types. Here, the T-cell and tumour cell profiles are the same for each value of $k_{3}$ (i.e. $T: m=1$ ). These results illustrate that alterations in both ligand and receptor mechanisms result in the increased sensitivity of the T-cells towards FasL, and cause a down-regulation of the immune response after MMP inactivation. 

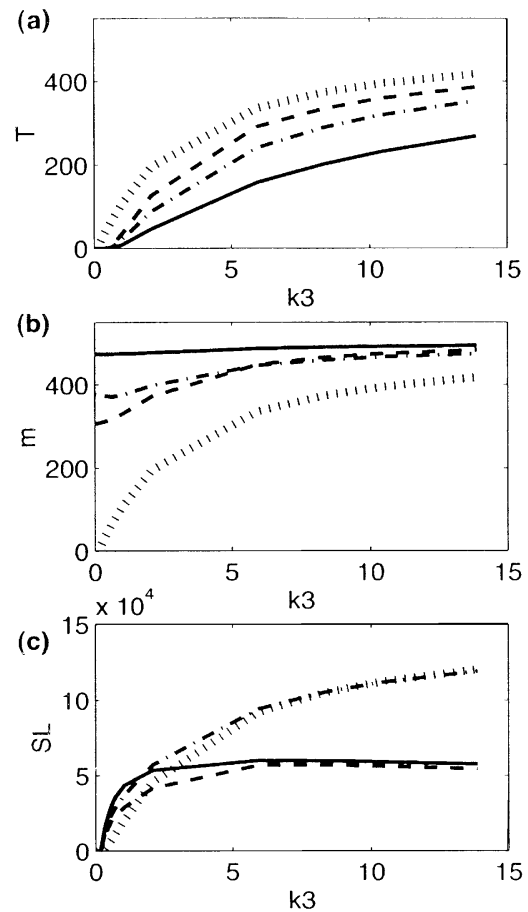

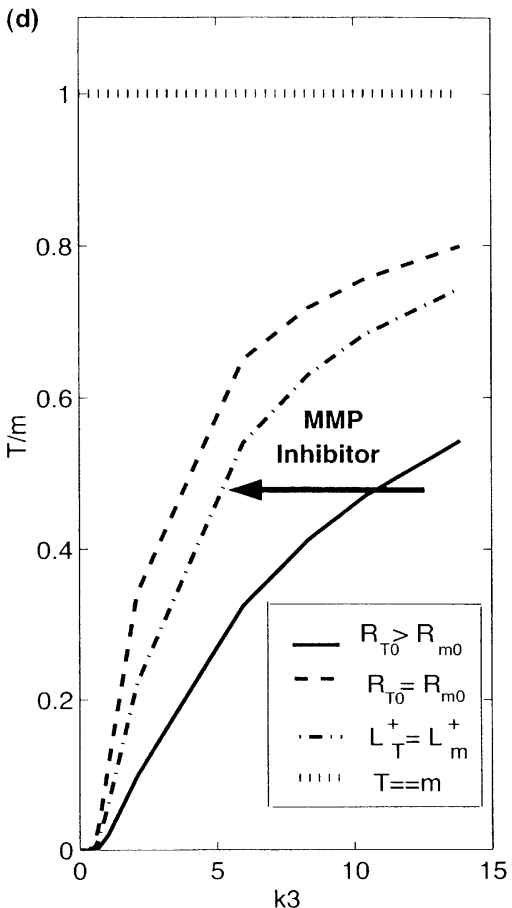

Fig. 4. Solutions of the model (1a)-(1g) after $48 \mathrm{~h}$ for increasing values of $k_{3}$, the rate constant which controls the MMP-mediated cleavage of FasL into sFasL. Increasing $k_{3}$ corresponds to a decrease in metalloproteinase inhibitor. As $k_{3}$ increases, the densities of (a) T-cells ( $T$ ), (b) tumour cells $(m)$, and (c) soluble ligand $\left(S_{L}\right)$, increases. In (d), we show the change in the T:m ratio as $k_{3}$ varies. In (i), (ii) the basic model is solved; in (iii), (iv) the $L_{T}$ equation is changed to give constitutive expression of FasL in T-cells (as in legend to Fig. 3). The initial conditions are: (i), (iii) $R_{T}(0)=10^{4}$ molecule/cell, $R_{m}(0)=10^{3}$ molecule/cell; (ii), (iv) $R_{T}(0)=R_{m}(0)=5 \times 10^{3}$ molecule/cell. The other parameters and initial conditions are as in Fig. 2. We observe that decreasing $k_{3}$ causes a decrease in the $T: m$ ratio. This predicts that metalloproteinase inhibitors cause T-cells to undergo apoptosis more rapidly than tumour cells. This can be explained by (a), (b): varying $k_{3}$ has the greatest effect on the level of T-cells. For example, varying $k_{3}$ from 5.9 to $13.8 \mathrm{~h}^{-1}$, increases the density of T-cells by $69 \%$, and tumour cells by $1.5 \%$ (solid line).

\section{Discussion}

In this paper, we have developed a mathematical model based on tumour cell-lymphocyte interaction and the cell surface expression of Fas and FasL, and have also extended the model to include the involvement of the MMP catalyzed soluble degradation product of the ligand (sFasL). To our knowledge, this is the first mathematical study of the FasL/Fas system and its role in tumour-immune interactions. The key features in the model are the constitutive expression and engagement of membrane bound FasL on tumours with Fas expressed on the surface of activated lymphocytes, and the down-regulation of the cell surface Fas in tumours. We predict that the production of soluble forms of Fas and FasL will lead to down-regulation of the immune response by neutralising the Fas ligand or receptor. Moreover, the inclusion of MMP inactivation in the model results in increased transmembrane FasL and leads to a higher rate of Fas-mediated apoptosis for lymphocytes than for tumour cells. 
Most tumour cell types constitutively express the Fas ligand and there is growing evidence to suggest that these FasL expressing tumour cells can actively kill Fas positive lymphocytes by apoptosis. This phenomenon has now been demonstrated not only from tumour cells derived from lymphoid tissues [37,38] but also in a wide range of human solid tumours and metastatic lesions including cancers of the colon [39], esophagus [40], kidney [41,42], pancreas [43], brain [44], head and neck [45], breast [46], ovary [47] and malignant pleural effusions [48]. The latter report includes work of Zeronski, who examined the expression of Fas/FasL and the incidence of apoptosis in tumour cells as well as tumour associated lymphocytes in 23 malignant pleural effusions obtained from patients with various metastatic solid tumours. These tumours were found to express FasL, whereas tumour-associated lymphocytes expressed Fas, but little FasL. Few tumour cells were apoptotic, whereas many tumour associated lymphocytes were undergoing apoptosis, and were also functionally deficient. Thus, it would appear that the Fas/FasL pathway contributes, at least in part, to the demise of tumour associated lymphocytes in a number of primary human solid cancers and in malignant effusions derived from a variety of metastatic tumours.

Not all patients express tumour membrane-bound FasL $[49,50]$ and the constitutive expression of this molecule even within an individual tumour appears to be heterogeneous [51]. Another complementary mechanism, that is also likely to be operational in vivo, is the well-known phenomenon of AICD, where the induction of FasL on the surface of T-cells after an immune response can result in lymphocyte suicide and/or fratricide [52]. Both the tumour FasL counterattack and AICD mechanisms have been suggested to be operational in some tumours [42,53] although the relative contribution of these two mechanisms to the demise of tumour associated lymphocytes in other solid tumours requires investigation. Nevertheless, it would be possible to enhance the model framework described in the present paper to include AICD. This would allow the study of a uniform in vivo concept in which both models could be operational under different conditions.

Treatment of activated T-cells [54] or tumour cell lines [55] with MMP inhibitors in vitro leads to the accumulation of membrane-bound FasL and a decrease in soluble FasL, resulting in apoptosis of Fas sensitive cell lines. Recently, it has been demonstrated that recombinant FasL can be efficiently cleaved in vitro by MMP-7 (matrilysin) but not MMP-2 (gelatinase A) or MMP9 (gelatinase B), and that MMP-7 can also decrease transmembrane FasL cell surface levels [16]. Thus, both tumour cells and activated lymphocytes in the presence of broad spectrum MMP inhibitors should, in principle, undergo Fas/FasL induced suicide. However, activated lymphocytes express high levels of Fas receptor and, although these cells can be initially resistant to Fas/ FasL suicide, during the course of an immune response lymphocytes become highly sensitive to Fas-mediated apoptosis [5]. In contrast, the majority of tumour cell types down-regulate the Fas receptor and are intrinsically resistant to Fas-mediated apoptosis [56-58]. Even tumours such as pancreatic cancer, that express both Fas receptor and ligand, are resistant to Fas-mediated apoptosis because an intracellular signal transduction inhibitor is up-regulated in this tumour type [43].

Most tumours are characterized by altered MMP activity, either expressed by tumour cells or, more often, from adjacent stromal cells (i.e. macrophages and fibroblasts). High levels of activated MMPs have been demonstrated in tumours that are more invasive and/or metastatic [59,60] and play a role in late stage tumour progression. However, unlike other MMPs, matrilysin is 
already expressed in the early stages of human intestinal cancer [61]. Thus, the formation of sFasL by MMP-7 cleavage, subsequent down-regulation of membrane bound Fas receptor, and desensitization of tumour cells to Fas signals may be early events in carcinogenesis. These possibilities require experimental verification in other tumour types.

Lymphocytes may also utilize several other apoptotic mechanisms under different conditions. For example, the $\mathrm{Ca}^{2+}$-dependent perforin-gramzyme mechanism and the more recently discovered TRAIL receptor system $[62,63]$ may also play a role in vivo. The relative contribution that these systems make in a variety of human non-lymphoid malignancies to the overall tumour celllymphocyte conflict is unknown and, for simplicity, has been neglected in the present study. Moreover, in addition to its role in triggering apoptosis, FasL appears to have several other functions. It may also act as an autocrine growth factor by promoting cell cycle progression and tumour cell survival, as demonstrated in glioma cells [64]. In contrast, enforced FasL expression in tumour cells and tissue grafts can elicit neutrophilic inflammation and rejection in some animal model systems $[65,66]$. The precise conditions that tilt the FasL reaction towards apoptosis/cell cycle progression as opposed to an inflammatory response remain unclear, although it has recently been demonstrated that transfected tumour cells expressing sFasL can suppress the inflammatory response and appears to protect tumour cells from this type of injury in vivo [67].

The clinical implications of the model predictions described in this paper are two-fold. Firstly, elevated levels of MMPs in tumours and their association with invasion and angiogenesis have led to the development of broad spectrum MMP inhibitors as potential therapeutic agents, and several compounds are undergoing clinical trials in cancer patients [68]. Such therapies could be compromised by the predicted adverse effect of MMP inactivation on FasL and lymphocyte apoptosis. On the other hand, MMP-7 inactivation would effectively lead to decreased levels of sFasL and this downregulation may abrogate the suppressive effect of the soluble ligand on neutrophilic inflammation and could result in tumour regression. Secondly, many tumour cell types are resistant to Fas-mediated apoptosis and these cells often have the capability of killing activated lymphocytes via expression of FasL. We suggest, therefore, that the Fas/FasL system may have an important impact on the outcome of numerous ongoing immunotherapeutic trials based upon, for example, antigenic peptide presentation, costimulation and activation of lymphocytes/dendritic cells, since the final common pathway of all these approaches is the transduction of death signals within the tumour cell.

\section{Acknowledgements}

This work was supported by SHEFC Research Development Grant 107, Cancer Research Wales, and EPSRC (earmarked studentship to S.D.W. and advanced research fellowship to J.A.S.).

\section{References}

[1] E.J. Fuchs, P. Matzinger, Is cancer dangerous to the immune system? Sem. Immunol. 8 (1996) 271.

[2] A.K. Abbas, C.A. Janeway, Immunology: Improving on nature in the twenty-first century, Cell 100 (2000) 129.

[3] C.C. Liu, L.H.Y. Young, J.D.E. Young, Lymphocyte-mediated cytolysis and disease, New Eng. J. Med. 335 (1996) 1651. 
[4] S. Chouaib, C. Asselin-Paturel, F. Mami Chouaib, A. Caignard, J.Y. Blay, The host-tumour immune conflict: from immunosuppression to resistance and destruction, Immunol. Today 18 (1997) 493.

[5] M. Irmler, M. Thorne, M. Hahne, P. Schneider, K. Hofman, V. Steiner, J.L. Bodmer, M. Schroter, K. Burns, C. Mattman, D. Rimoldi, L.E. French, J. Tschopp, Inhibition of death receptor signals by cellular FLIP, Nature 388 (1997) 190.

[6] J. O'Connell, M.W. Bennett, G.C. O'Sullivan, J.K. Collins, F. Shanahan, The Fas counterattack: cancer as a site of immune privilege, Immunol. Today 20 (1999a) 46.

[7] D.H. Lynch, F. Ramsdell, M.R. Alderson, Fas and Fas-L in the homeostatic regulation of immune responses, Immunol. Today 16 (1995) 569.

[8] T.A. Ferguson, T.S. Griffiths, A vision of cell death: insights into immune privilege, Immunol. Rev. 156 (1997) 167.

[9] I. Cascino, G. Fiucci, G. Papoff, G. Ruberti, Three functional forms of the human apoptosis-inducing Fas molecule are produced by alternative splicing, J. Immunol. 154 (1995) 2706.

[10] T. Suda, H. Hashimoto, M. Tanaka, T. Ochi, S. Nagata, Membrane Fas ligand kills human peripheral blood T-lymphocytes and soluble Fas ligand blocks the killing, J. Exp. Med. 186 (1997) 2045.

[11] M. Shibakita, M. Tachibana, D.K. Dhas, T. Kotoh, S. Kinugasa, H. Kubota, R. Masunaga, N. Magasue, Prognostic significance of Fas and Fas ligand expressions in human esophageal cancer, Clin. Cancer Res. 5 (1999) 2464.

[12] H. Das, T. Koizumi, T. Sugimoto, S. Chakraborty, T. Ichimura, K. Hesagawa, R. Nishimura, Quantitation of Fas and Fas ligand gene expression in human ovarian, cervical and endometrial carcinomas using real-time quantitive RT-PCR, Brit. J. Cancer 82 (2000) 1682.

[13] M. Tanaka, T. Suda, K. Haze, N. Nakamura, K. Sato, F. Kimura, K. Motoyoshi, M. Mizuki, S. Tagawa, S. Ogha, K. Hatake, A.H. Drummond, S. Nagata, Fas ligand in human serum, Nat. Med. 2 (1996) 317.

[14] G.P. Midas, Y. Shen, L.B. Owen-Schaub, Elevated soluble Fas (sFas) levels in non-hematopoietic human malignancy, Cancer Res. 56 (1996) 3370.

[15] M. Tanaka, T. Itai, M. Adachi, S. Nagata, Down-regulation of Fas ligand by shedding, Nat. Med. 4 (1998) 31.

[16] W.C. Powell, B. Fingleton, C.L. Wilson, M. Boothby, L.M. Matrisian, The metalloproteinase matrilysin proteolytically generates active soluble Fas ligand and potentiates epithelial cell apoptosis, Curr. Biol. 9 (1999) 1441.

[17] J.A. Adam, N. Bellomo, A Survey of Models for Tumour-Immune System Dynamics, Birkhäuser, Boston, 1996.

[18] A. Albert, M. Freedman, A.S. Perelson, Tumours and the immune system: the effects of a tumour growth modulator, Math. Biosci. 50 (1980) 25.

[19] J.A. Adam, The dynamics of growth factor-modified immune response to cancer growth: one dimensional models, Math. Comp. 17 (1993) 83.

[20] D. Kirshner, J.C. Panetta, Modelling immunotherapy of the tumour-immune interaction, J. Math. Biol. 37 (1998) 235.

[21] M.R. Owen, J.A. Sherratt, Pattern formation and spatiotemporal irregularity in a model for macrophage-tumour interaction, J. Theor. Biol. 189 (1997) 63.

[22] J.A. Adam, Effects of vascularization on lymphocyte/tumour cell dynamics: qualitative features, Math. Comp. 23 (1996) 1.

[23] V.A. Kuznetsov, Harpoon model for cell-cell adhesion and recognition of target cells by the natural killer cells, J. Theor. Biol. 180 (1996) 321.

[24] A. Yates, C. Bergmann, J. LeoVan Hemmen, J. Stark, R. Callard, Cytokine-modulated regulation of helper T-cell populations, J. Theor. Biol. 206 (2000) 539.

[25] J.A. Janeway, P. Travers, Immunology: the Immune System in Health and Disease, Current Biology, London, 1996.

[26] K.E. Hellstrom, I. Hellstrom, Tumours immunological escape of, in: P.J. Delves, I.M. Roitt (Eds.), Encyclopedia of Immunology, 2nd Ed., vol. 4, Academic Press, London, 1998, p. 2443.

[27] M.R. Owen, J.A. Sherratt, Modelling the macrophage invasion of tumours: effects on growth and composition, IMA J. Math. Appl. Med. Biol. 15 (1998) 165.

[28] T.S. Griffith, T. Brunner, S.M. Fletcher, D.R. Green, T.A. Ferguson, Fas ligand induced apoptosis as a mechanism of immune privilege, Science 270 (1995) 1189. 
[29] F. Vignaux, E. Vivier, B. Malissen, V. Depraetere, S. Nagata, P. Golstein, TCR/CD3 coupling to Fas-based cytotoxicity, J. Exp. Med. 181 (1995) 781.

[30] G. Bossi, G.M. Griffiths, Degranulation plays an essential part in regulating cell surface expression of Fas ligand in T-cells and natural killer cells, Nat. Med. 5 (1999) 90.

[31] T.L. Whiteside, H. Rabinowich, The role of Fas/Fas-L in immunosuppression induced by human tumours, Cancer Immunol. Immunother 46 (1998) 175.

[32] T. Suda, T. Takahashi, P. Golstein, S. Nagata, Molecular-cloning and expression of the Fas ligand, a novel member of the tumor-necrosis-factor family, Cell 75 (1993) 1169.

[33] D.A. Lauffenburger, J.J. Linderman, Receptors: models for binding, trafficking and signalling, Oxford University, Oxford, 1993.

[34] R. Watanabe-Fukunaga, C.I. Brannan, N. Itoh, S. Yonehara, N.G. Copeland, N.A. Jenkins, S. Nagata, The cDNA structure, expression, and chromosomal assignment of the mouse Fas antigen, J. Immunol. 148 (1992) 1274.

[35] P.A. Kiener, P.M. Davis, B.M. Rankin, S.J. Klebanoff, J.A. Ledbetter, G.C. Starling, W.C. Liles, Human monocytic cells contain high levels of intracellular Fas ligand: rapid release following cellular activation, J. Immunol. 159 (1997) 1594.

[36] S.D. Webb, J.A. Sherratt, A perturbation problem arising from the modelling of soluble Fas ligand in tumour immunology, Math. Comp. Modelling (2002) submitted.

[37] A. Zeytun, M. Hassunch, M. Nagarkatti, P.S. Nagarkatti, Fas-Fas ligand-based interactions between tumour cells and tumour specific cytotoxic T lymphocytes: a lethal two-way street, Blood 90 (1997) 1952.

[38] A. Villunger, A. Egle, I. Marchitz, M. Kos, G. Bock, H. Ludwig, S. Geley, R. Kofler, R. Greil, Constitutive expression of Fas (Apo-1/CD95) ligand on multiple myeloma cells: a potential mechanism of tumour-induced suppression of immune surveillance, Blood 90 (1997) 12.

[39] K. Shiraki, N. Tsaji, N. Shioda, K.J. Isselbacker, H. Takahashi, Expression of Fas ligand in liver metastases of human colonic adenocarcinomas, Proc. Nat. Acad. Sci. 94 (1997) 6420.

[40] C. Gratas, Y. Tohma, C.B. Barnes, P. Taniere, P. Hainaut, H. Ohgaki, Up-regulation of Fas (APO-1/CD95) ligand and down-regulation of Fas expression in human esophageal cancer, Cancer Res. 58 (1998) 2057.

[41] G. Cardi, J.A. Heaney, A.R. Schned, M.S. Ernstoff, Expression of Fas (Apo-1/CD95) in tumour-infiltrating and peripheral blood lymphocytes in patients with renal cell carcinoma, Cancer Res. 58 (1998) 2078.

[42] R.G. Uzzo, P. Rayman, V. Kolenko, P.E. Clark, T. Bloom, A.M. Ward, L. Molto, C. Tannenbaum, L.J. Worford, R. Bukowski, R. Tubbs, E.D. Hsi, N.H. Bander, A.C. Novick, J.H. Finke, Mechanisms of apoptosis in T cells from patients with renal cell carcinoma, Clin. Cancer Res. 5 (1999) 1219.

[43] H. Ungefroren, M. Voss, M. Jansen, C. Roeder, D. Henne-Bruns, B. Kramer, H. Kalthoff, Human pancreatic adenocarcinomas express Fas and Fas-L yet are resistant to Fas-mediated apoptosis, Cancer Res. 58 (1998) 1741.

[44] M. Weller, P. Kleihnes, J. Dichgans, H. Ohgaki, CD95 ligand: lethal weapon against malignant glioma, Brain Pathol. 8 (1998) 285.

[45] B.R. Gastman, Y. Atarashi, T.E. Reichert, T. Saito, L. Balkir, H. Rabinowich, T.L. Whiteside, Fas ligand is expressed in human squamous carcinomas of the head and neck and its promotes apoptosis of $\mathrm{T}$ lymphocytes, Cancer Res. 20 (1999) 5356.

[46] J. O’Connell, M.W. Bennett, G.C. O’Sullivan, J. O'Callaghan, J.K. Collins, F. Shanahan, Expression of Fas (CD95/APO-1) ligand by human breast cancers: significance for tumour immune privilege, Clin. Diagnostic Lab. Immunol. 6 (1999b) 457.

[47] S. Munakata, T. Enomoto, M. Tsujimoto, Y. Otsuki, H. Moiva, H. Kanno, K. Aozasa, Expression of Fas ligand and other apoptosis-related genes and their prognostic significant in epithelial ovarian neoplasms, Brit. J. Cancer $82(2000) 446$.

[48] R.B. Herberman, T.L. Whiteside, Summary of the international cancer microenvironment from meeting held in Pittsburgh, Pennsylvania, Cancer Res. 60 (2000) 1465.

[49] D.B. Chappell, T.Z. Zaks, S.A. Rosenberg, N.P. Restifo, Human melanoma cells do not express Fas (Apo-1/ CD95) ligand, Cancer Res. 59 (1999) 59.

[50] P. Terheyden, C. Siedel, A. Merkel, E. Kampgen, E.B. Brocker, J.C. Becker, Predominant expression of Fas (CD95) ligand in metastatic melanoma revealed by longitudinal analysis, J. Invest. Dermatol. 112 (1999) 899. 
[51] M.W. Bennet, J. O'Connell, G.C. O'Sullivan, C. Brady, D. Roche, J.K. Collins, F. Shanahan, The Fas counterattack in vivo: apoptotic depletion of Tumour-infiltrating lymphocytes associated with Fas ligand expression by human esophageal carcinoma, J. Immunol. 160 (1998) 5669.

[52] M.R. Alderson, T.W. Tough, T.D. Smith, S. Braddy, B. Falk, K.A. Schooley, R.G. Goodwin, C.A. Smith, F. Ramsdell, D.H. Lynch, Fas ligand mediates activation-induced apoptosis cell death in human lymphocytes, J. Exp. Med. 181 (1995) 71.

[53] T.Z. Zaks, D.B. Chappell, S.A. Rosenberg, N.P. Restifo, Fas-mediated suicide of tumour-reactive T-cells following activation by specific tumour: selective rescue by caspase inhibition, J. Immunol. 162 (1999) 3273.

[54] N. Kayagaki, A. Kawasaki, T. Ebata, H. Ohmoto, S. Ikeda, S. Inove, K. Yoshino, K. Okumura, H. Yagita, Metalloproteinase-mediated release of human Fas ligand, J. Exp. Med. 182 (1995) 1777.

[55] N. Mitsiades, V. Poulaki, A. Leone, M. Tsokos, Fas-mediated apoptosis in Ewings Sarcoma cell lines by metalloproteinase inhibitors, J. Nat. Cancer. Inst. 91 (1999) 1678.

[56] K. Higaki, H. Yano, M. Kojiro, Fas antigen expression and its relationship with apoptosis in human hepatocellular carcinoma and non-cancerous tissues, Amer. J. Path. 149 (1996) 429.

[57] C.A. Maggi, Therapeutic opportunities from the pharmacological manipulation of the Fas system, Pharmacol. Res. 38 (1998) 1.

[58] M. Mottolose, S. Buglioni, C. Bracalenti, M.A. Cardarelli, L. Ciabocco, D. Gi-annarelli, C. Botti, P.G. Natali, A. Concetti, M. Venanzi, Prognostic relevance of altered Fas (CD95)-system in human breast cancer, Int. J. Cancer Pred. Oncol. 89 (2000) 127.

[59] A.F. Chambers, L.M. Matrisian, Changing views of the role of matrix metalloproteinases in metastasis, J. Nat. Cancer Inst. 89 (1997) 1260.

[60] A.R. Nelson, B. Fingleton, M.L. Rothenberg, L.M. Matrisian, Matrix metalloproteinases: biological activity and clinical applications, J. Clin. Oncol. 18 (2000) 1135.

[61] B. Fingleton, K.J. Heppner-Goss, H.C. Crawford, L.M. Matrisian, Matrilysin in early stage intestinal tumorigenesis, APMIS 107 (1999) 102.

[62] J.A. Trapani, J. Davis, V.R. Sutton, M.J. Smyth, Proapoptotic functions of cytotoxic lymphocyte granule constituents in vitro and in vivo, Curr. Op. Immunol. 12 (2000) 323.

[63] H. Walczak, P.H. Krammer, The CD95 (APO-1/Fas) and the TRAIL (APO-2L) apoptosis systems, Exp. Cell Res. 256 (2000) 58.

[64] H. Shinohara, H.I. Yagita, Y. Kawa, N. Oyaizu, Fas drives cell cycle progression in glioma cells via extra cellular signal regulated kinase activation, Cancer Res. 60 (2000) 1766.

[65] P.R. Walker, P. Saas, P.Y. Dietrich, Tumour expression of Fas ligand (CD95L) and the consequences, Curr. Op. Immunol. 10 (1998) 564.

[66] N.P. Restifo, Not so Fas: re-evaluating the mechanisms of immune privilege and tumour escape, Nat. Med. 6 (2000) 493.

[67] A.M. Hohlbaum, S. Moe, A. Marshak-Rothstein, Opposing effects of transmembrane and soluble Fas ligand expression on inflammation and tumour cell survival, J. Exp. Med. 191 (2000) 1209.

[68] G. Deplanque, A.L. Harris, Anti-angiogenic agents: clinical trial design and therapies in development, Eur. J. Cancer 36 (2000) 1713. 